\title{
ROUTINE ANTENATAL SYPHILIS SCREENING IN SOUTH WEST NIGERIA- A QUESTIONABLE PRACTICE
}

\author{
*Adesina Olubukola and *Oladokun Adesina
}

*Department of Obstetrics and Gynaecology, College of Medicine, University of Ibadan, Nigeria.

Correspondence:

Dr. Adesina Olubukola

Department of Obstetrics \& Gynaecology,

College of Medicine,

University of Ibadan, Nigeria.

bukiadewole@yahoo.com,

bukiadewole@hotmail.com

Phone: 234-803-3486836

\begin{abstract}
Background: Untreated maternal syphilis is strongly associated with adverse birth outcomes, especially in women with high titre syphilis. The WHO recommends routine serological screening in pregnancy. Some workers have advised a reappraisal of this practice, having demonstrated low sero-prevalence in their antenatal population. In view of this, the aim of this study was to determine the seroprevalence of syphilis in the antenatal population presenting at a major hospital in south-west Nigeria.

Methods: This was a cross sectional study of healthy pregnant Nigerian women attending Adeoyo Maternity Hospital in the capital of Oyo State. The case record of every pregnant woman presenting for their first antenatal clinic visit over a 4-month period (September $1^{\text {st }}$ to December $31^{\text {st }}$ 2006) was reviewed.

Results: During the study period, two thousand six hundred and seventy-eight women sought antenatal care. Three hundred and sixty-nine women $(369 ; 13.4 \%)$ had incomplete records and were excluded from analysis. The records of the 2,318(86.6\%) women with adequate records were subsequently reviewed. The mean age of the women was 27.4 years $( \pm 5.34)$ and the mean gestational age 26.4 weeks ( \pm 6.36$)$. The modal parity was 0 . Only three patients were found to be reactive for syphilis giving a prevalence of $0.13 \%$. Conclusion: The sero- prevalence value in this study is quite low and may justify the call to discontinue routine antenatal syphilis screening. However, a more rigorous screening program using diagnostic tests with higher sensitivity maybe necessary before jettisoning this traditional aspect of antenatal care.
\end{abstract}

Keywords- Pregnancy; syphilis; prevalence; serology; screening

\section{INTRODUCTION}

Syphilis, caused by infection with Treponema pallidum, is a muco-cutaneous sexually transmitted infection (STI) with high infectivity in the early stages. It may also be passed transplacentally from the ninth week of gestation onwards ${ }^{1}$. The basic pathology in all stages is vasculitis $^{2}$. Untreated maternal syphilis is strongly associated with adverse birth outcomes, especially in women with high titre syphilis (HTS) observed in earlier stages of the infection. These adverse birth outcomes include an increased incidence of stillbirths, low birth weight and premature live births compared with uninfected women ${ }^{3}$. An increased incidence of spontaneous abortions after 20 weeks of gestation has also been reported ${ }^{4}$. Congenital syphilis infection results in fetal or perinatal death in $40 \%$ of affected pregnancies, as well as disease complications in surviving newborns, including central nervous system abnormalities; deafness; multiple skin, bone, and joint deformities; and haematological disorders ${ }^{5}$.

The World Health Organization (WHO) recommends serological screening in pregnancy and treatment with injectable penicillin, including the partner, as a routine part of antenatal care ${ }^{6}$. Ideally, this screening should be done during the first trimester or at the first antenatal visit of the woman and again early in the third trimester, even in low- prevalence populations. There is observational evidence that suggests that the universal screening of pregnant women decreases the proportion of infants with clinical manifestations of syphi- 
lis infection and those with positive serologies ${ }^{5}$. Indeed, the benefits of screening all pregnant women for syphilis infection substantially outweigh potential harms ${ }^{5,7}$. Some workers have however advised a reappraisal of the practice of universal antenatal screening for syphilis having demonstrated low sero-prevalence in their antenatal population. They questioned the cost-effectiveness of this practice given its low yield $^{8}$.

\section{Objectives}

In view of these observations, the aim of this study was to determine the sero-prevalence of syphilis in a group of pregnant Nigerian women presenting at a secondary health care institution in south-west Nigeria.

\section{MATERIALS AND METHODS}

This is a cross sectional study of apparently healthy pregnant Nigerian women attending Adeoyo Maternity Hospital, a secondary health centre in Ibadan, the capital of Oyo State in the South-West of Nigeria. The case record of every pregnant woman presenting for their first antenatal clinic visit over a 4-month period (September $1^{\text {st }}$ to December $31^{\text {st }} 2006$ ) was reviewed. Data was collected by the means of a prepared proforma. These data included selected demographic and obstetrics information. Information on the result for screening for syphilis was also extracted. Women with incomplete data were excluded from the study. Ethical approval was obtained from the University College Hospital/ University of Ibadan institutional ethical review committee.

\section{Laboratory diagnosis of Syphilis}

Venous blood $(5 \mathrm{ml})$ was collected from the antecubital vein of each woman into sterile tubes. The blood was allowed to retract and then centrifuged, and the serum was obtained and stored at $-20^{\circ} \mathrm{C}$ until tested. All serum samples, test antigens and control samples were brought to room temperature $\left(26^{\circ} \mathrm{C}\right)$ and tested by the Venereal Disease Research Laboratory test using a VDRL test kit (Cal-Tech Diagnostics Inc., Chino, California, USA).

\section{Statistical Analyses}

Data were summarized as means \pm standard deviation, percentages and mode and analysed by independent T-test. Data entry and analysis was done using SPSS statistical package, version 15.0. P values less than 0.05 were considered significant.

\section{RESULTS}

From September $1^{\text {st }}$ to December $31^{\text {st }} 2006,2678$ two thousand six hundred and seventy- eight $(2,678)$ women sought antenatal care, at Adeoyo Maternity
Hospital, Yemetu, Ibadan. Three hundred and sixtynine women $(369 ; 13.4 \%)$ had incomplete records and were excluded from further analysis. The records of the 2,318 women with adequate records were subsequently reviewed for the sero-prevalence of syphilis. The mean age of the women was 27.4 years $( \pm 5.34)$ and they presented at a mean gestational age of 26.4 weeks $( \pm 6.36)$. Most of the patients were in the age group 20 to 34 years. The modal parity was 0 . Slightly over half of the patients were in their second trimester of pregnancy. Only three patients were found to be reactive for syphilis giving a prevalence of $0.13 \%$. There was no record of confirmatory tests in the patients' records.

\section{DISCUSSION}

The sero-positivity on screening of $0.13 \%$ found in this study is quite low. Other workers in Ibadan at various times have reported progressively lower values on screening. Oyelese et al, in 1990 reported 2.3\%, Adewole et al, in 1997 reported 1.55\% while Obisesan et al, in 1999 reported 1.1\%,8,10. In Enugu, southeastern Nigeria screening values of $3.06 \%, 1.3 \%$ and $0.125 \%$ have been reported by successive workers ${ }^{11,12,13}$.

Sero-prevalence during pregnancy is generally low in developed countries: it ranges from $0.02 \%$ in Europe to a nationwide incidence rate of 2.4 per 100,000 persons for primary and secondary cases of syphilis infection in the United States. These reports from Nigeria suggest a gradual reduction in the prevalence of syphilis in these obstetric populations. As a result of these, Obisesan et al in 1999 suggested that screening for syphilis was no longer cost effective and recommended that the practice be discouraged. However, Taiwo et al also working in south -west Nigeria, more recently reported a prevalence of $9.9 \%$ and strongly advised that the practice continue ${ }^{14}$. Watson-Jones et al working in Tanzania also noted wide variations in the values reported at various sites in that country and attributed these to basic misunderstandings about the testing procedure among the health workers and a lack of refresher training and quality assurance of program activities $^{3}$. It may thus be premature to discourage the practice of screening for syphilis without conclusively confirming these low rates in various obstetric populations in the country, using tests with higher sensitivity.

Traditionally, screening for syphilis infection is a 2-step process that involves an initial non-treponemal test e.g. Venereal Disease Research Laboratory(VDRL) followed by a confirmatory treponemal test e.g. T. pallidum particle assay (TP-PA) ${ }^{5}$. There was no record of the patients returning for confirmatory tests. 
Obisesan and Ahmed, in their study, had reported low rates of repeat testing and no confirmatory tests being carried out ${ }^{8}$. This buttresses the call for same day on-site testing and treatment, as African women are apt not to return for follow-up visits ${ }^{3}$. All positive tests, whether cardiolipin or treponemal antigen based, should preferably be confirmed with a different method from the initial test. Where confirmatory tests are not easily available, treatment should be initiated as delay in treatment is much more deleterious than not getting confirmation of tests ${ }^{2}$. The health care provider must also note that screening is not totally innocuous and potential harms of screening may include opportunity costs to the clinician and patient (time, resources, etc.) and false-positive results which may lead to stress, labeling, and further workup ${ }^{5}$. Falsepositive results may result from pregnancy itself and other conditions such as tuberculosis and malaria ${ }^{5}$, many conditions quite common in the tropical environment where this study was performed

While the non-treponemal tests are useful for screening infectious syphilis, they will fail to diagnose many primary and late latent/late syphilis as the sensitivity is $44-76 \%{ }^{2}$. Prozone phenomenon in secondary syphilis may give false negative results using undiluted serum can occur ${ }^{15}$. These are possible factors that must be considered before accepting the low values reported in this study and/ or discarding the tradition of syphilis testing. This is important because VDRL/RPR is still commonly used as a screening test in many of our institutions, as this one where this survey was carried out, because of cost and ease of performance. If a single test is to be used, the TPPA/TPHA (T. pallidum haemagglutination assay) or treponemal enzyme immunoassay (EIA) is preferable to the RPR/VDRL as it will diagnose almost all stages of syphilis except for primary syphilis. For screening, the sensitivities of EIA and TPHA/TPPA are 82-100\% and 85-100\% with specificity of $97-100 \%$ and $98-100 \%$, respectively ${ }^{2}$. Indeed, investing in these diagnostic tests may assist care providers in identifying more cases of syphilis in pregnancy. New screening tests currently being studied for use in pregnant women and infants include: IgM immunoblotting and Polymerase Chain Reaction (PCR) assay of serum and cerebrospinal uid for central nervous system infection in infants, placenta histopathology, and umbilical cord blood testing ${ }^{5,16}$. These may however be beyond the meager resources available in most of our health institutions including that where this study was done.

It is thus obvious that before totally discarding the practice of screening for syphilis, it will be necessary to screen this obstetric population with test kits of higher sensitivity, such as those described above. This is because of the serious nature of the morbidities associated with untreated maternal syphilis. In addition, it is important to recognize that syphilis is capable of re-emerging in populations extremely rapidly when prevention efforts decline or collapse ${ }^{17,18}$. This is another argument to support the antenatal practice of screening for syphilis. In conclusion, the seroprevalence in this obstetric population was quite low and there may be justification for the call to discontinue the routine antenatal screening. However, a more rigorous screening program using diagnostic tests with higher sensitivity maybe necessary before totally jettisoning this traditional aspect of antenatal care.

\section{REFERENCES}

1. Lewis D.A. and Young H. Syphilis. Sex Transm Inf. 2006; 82: iv13-iv15

2. Goh B.T. Syphilis in adults Sex Transm Infect 2005; 81:448-452

3. Watson-Jones D., Oliff M., Terris-Prestholt F. et al., Antenatal syphilis screening in sub-Saharan Africa: lessons learned from Tanzania. Trop Med Int Health 2005; 10: 934-943.

4. McDermott J., Steketee R., Larsen S. and Wirima J. Syphilis-associated perinatal and infant mortality in rural Malawi. Bull World Health 1993; 71: $773-780$.

5. U.S. Preventive Services Task Force. Screening for Syphilis Infection: Recommendation Statement. Ann Fam Med 2004; 2: 362-365.

6. World Health Organisation. Guidelines for the Management of Sexually Transmitted Infections. (2001) World Health Organisation, Geneva.

7. Marx R., Aral S.G., Rolfs R.T. et al., Current Trends Congenital Syphilis_-United States, 1983-1985. MMWR. 1986; 35: 625-628

8. Obisesan K.A. and Ahmed Y. Routine antenatal syphilis screening- a case against. Afr J. Med. Med. Sci. 1999; 28: 185-187

9. Oyelese A.O., Asuzu M.C., and Osoba A.O. Pattern of reactive groups attending the University College Hospital, Ibadan, (1976- 1985). Afr J. Med. Med. Sci. 1990; 19: 163-166.

10. Adewole I.F., Fawole R.O. and Babarinsa I.A. The value of antenatal screening for syphilis in Ibadan. Niger Med Pract. 1997; 34: 39-41 
11. Gini P.C., Chukudebelu W.O. and Njoku-Obi A.N. Antenatal screening for syphilis at the University of Nigeria teaching hospital, Enugu, Nigeria- a six year survey. Int J Gynaecol Obstet. 1989; 29: 321-324.

12. Ozumba U.C., Oshi D.C., Nwokeji C.M. and Anya S.E. Trends in seroreactivity for syphilis among pregnant Nigerian women. Sex Transm Inf 1999; 75: 120-123

13. Ikeme A.C. and Okeke T.C. The prevalence of VDRL as routine test in pregnant women: a critical study. Niger J Clin Pract. 2006; 9: 65-7.

14. Taiwo S.S., Adesiji Y.O. and Adekanle D.A. Screening for syphilis during pregnancy in Nigeria: a practice that must continue. Sex Transm Infect 2007; 83: 357-358.
15. Gwanzura L., Latif A. and Bassett M., et al. Syphilis serology and HIV infection in Harare, Zimbabwe. Sex Transm Infect 1999; 75: 426-30

16. Nelson H.D., Glass N., Huffman L. et al., Screening for syphilis: a brief update for the U.S. Preventive Services Task Force. Rockville, MD, Agency for Healthcare Research and Quality. 2004. Available at: www.preventiveservices.ahrq.gov.

17. Imperato P.J. The current syphilis epidemic in New York State. New York State Journal of Medicine 1991; 91: 521-522.

18. Borisenko K.K., Tichonova L.I. and Renton A.M. (1999) Syphilis and other sexually transmitted infections in the Russian Federation. Int J STD AIDS 1999; 10: 665-668. 